\title{
Modelagem Matemática de problemas logísticos: discutindo o processo de entrega de urnas para eleição de Reitor do IFES
}

\author{
Mathematical Modeling of logistic problems: discussing the urns delivery process for \\ election of IFES Rector
}

\begin{abstract}
Lauro Chagas e Sá
Instituto Federal de Educação, Ciência e Tecnologia do Espírito Santo (IFES) Coordenadoria de Logística, Viana, ES, Brasil lauro.sa@ifes.edu.br
\end{abstract}

Lara Sossai Corrêa da Costa Instituto Federal de Educação, Ciência e Tecnologia do Espírito Santo (IFES) Coordenadoria de Logística, Viana, ES, Brasil larasossai@gmail.com

Bruna Zution Dalle Prane Instituto Federal de Educação, Ciência e Tecnologia do Espírito Santo (IFES) Coordenadoria de Matemática, Vitória, ES, Brasil bruna.prane@ifes.edu.br

\section{Informações do Artigo}

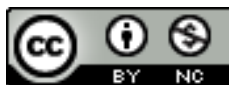

Histórico do Artigo

Submissão: 4 de setembro de 2018.

Aceite: 30 de outubro de 2018.

Palavras-chave

Pesquisa Operacional Teoria de Grafos Modelagem Matemática Teoria da Atividade

\section{Resumo}

O presente trabalho insere-se nos estudos sobre integração de componentes curriculares da Educação Profissional, a partir de estudos em Pesquisa Operacional, segundo a Teoria da Atividade de Leontiev (1978). Neste texto, o objetivo foi analisar um estudo de caso denominado "Entrega das urnas para Eleição de Reitor do IFES". O campo de investigação foi a disciplina de Pesquisa Operacional Aplicada à Logística, ofertada no Curso Superior de Tecnologia em Logística do IFES - Campus Viana, em 2017. Durante a disciplina, foi abordado o conteúdo de grafos, associado à logística. Para resolução dos problemas, os alunos dividiram-se em grupos e receberam fichas-contexto e uma tarefa a modelar. Optamos pela Modelagem Matemática porque a vemos como aliada da Educação Profissional. Ao analisar as produções, verificamos que alguns grupos entregaram trabalhos observando apenas a entrega das urnas, enquanto outros consideraram fatores externos, como jornada de trabalho dos servidores etc. Ou seja, por mais que os alunos tenham recebido o mesmo material didático e desenvolvido as mesmas tarefas, houve diferentes estratégias de resolução para a mesma atividade. Um dos fatores que está relacionado a essa intensidade de envolvimento na atividade é a motivação interna, influenciada pelo contexto histórico-cultural do sujeito (LEONTIEV, 1978). Com isso, concluímos que essa experiência apontou para um ensino emancipador, pois, a partir de um tema não-matemático, os alunos formularam e resolveram problemas, sendo responsáveis pela coleta de informações, simplificação das situações e realização da modelagem. Assim, esperamos que, com a emancipação dos alunos, alcancemos a emancipação de toda a classe trabalhadora (SAVIANI, 2017).

\section{Abstract}

The present work is part of the studies about integration of curricular components of Professional Education, based on studies in Operational Research, according to Leontiev's theory of activity (1978). In this text, the objective was to analyze a case study called "Delivery of the urns for the Election of IFES Rector". The research field was the discipline of Operational Research Applied to Logistics, offered in the Higher Course of Technology in Logistics of IFES - Campus Viana, in 2017. During the course, graphs were studied associated with logistics. To solve the 
problems, the students were divided into groups and received context sheets and a task to model. We opted for Modeling, since we see it as an ally of Professional Education. When analyzing the productions, we verified that some groups worked observing only the urns delivery, while others considered external factors, such as servers work hours, etc. That is, although the students received the same didactic material and developed the same tasks, there were different resolution strategies for the same activity. One of the factors that is related to this intensity of involvement in the activity is the internal motivation, influenced by the historical-cultural context of the subject (LEONTIEV, 1978). With this, we conclude that this experience pointed to an emancipatory teaching, because from a non-mathematical theme, students formulated and solved problems, being responsible for information gathering, simplification of situations and modeling. Thus, we hope that, with the emancipation of the students, we will achieve the emancipation of the entire working class (SAVIANI, 2017).

\section{Introdução}

A logística, como atividade, já está estabelecida no Brasil há aproximadamente seis décadas. Nesse contexto, este trabalho procura suscitar reflexões sobre como a Pesquisa Operacional (PO) pode favorecer a formação profissional do superior em logística, a partir de uma experiência de ensino à luz da Teoria da Atividade de Alexei Leontiev (1903-1979).

Segundo a Teoria da Atividade, as ações humanas são formas de relação do indivíduo com o mundo e são dirigidas por motivos e objetivos, agindo de forma intencional, por meio de ações planejadas (LEONTIEV, 1978). Nessa perspectiva, o contexto histórico-cultural de cada indivíduo influencia no seu aprendizado, na sua produção de conhecimento e, mais ainda, na sua atuação profissional. Isso porque, com o homem na vida adulta, o trabalho é atividade que guia seu desenvolvimento (LEONTIEV, 1978, apud FERUZZI, 2003).

O presente trabalho ${ }^{1}$ objetivou dar continuidade aos estudos sobre a integração de componentes curriculares da educação profissional de nível superior, a partir de estudos em Pesquisa Operacional, analisando um estudo de caso denominado "Entrega das urnas para Eleição de Reitor do IFES". Goldbarg e Luna (2005) definem a PO como modelos estruturados de forma lógica e amparados no ferramental matemático de representação, objetivando claramente a determinação das melhores condições de funcionamento para os sistemas representados. Reforçam, ainda, que os principais modelos de PO são chamados de programação matemática e formam uma das mais importantes variedades dos modelos quantitativos. Portanto, entende-se que a PO é abrangente e suas técnicas são de grande utilidade para a otimização de problemas como também para auxílio no processo de tomadas de decisão.

Para Moreira (2007), os modelos da PO visam a auxiliar na seleção da melhor maneira de aproveitar, nas organizações de produção, os recursos limitados existentes. De acordo com Wilde

\footnotetext{
${ }^{1}$ Este texto trata-se de um recorte da pesquisa de Iniciação Científica "Modelagem matemática de problemas logísticos: analisando atividades de Pesquisa Operacional à luz da Teoria de Leontiev", cadastrada no IFES sob $n^{\circ}$ PT00006600. Também se vincula ao EMEP - Grupo de Pesquisa em Educação Matemática e Educação Profissional (http://emep.ifes.edu.br).
} 
e Beightler (1967), a PO destaca-se, principalmente, devido à grande aplicabilidade encontrada na solução de problemas de otimização, agrupando diversas técnicas e algoritmos que tentam solucionar modelos quantitativos. A Associação Brasileira de Engenharia de Produção - ABEPRO (2011) caracteriza a Pesquisa Operacional como uma área de conhecimento, com as seguintes subáreas: Modelagem, Simulação e otimização; Programação matemática; Processos decisórios; e Processos estocásticos. Já para Goldbarg e Luna (2005), os principais modelos de PO são denominados de programação, de acordo com as técnicas utilizadas para a resolução de modelos matemáticos. Esses dividem-se em: problemas lineares, sendo que as variáveis são contínuas e apresentam comportamento linear; problemas inteiros, se existir alguma variável condicionada a assumir valores discretos; problemas não-lineares, quando exibem qualquer tipo de nãolinearidade.

A Pesquisa Operacional, que teve sua origem na Segunda Guerra Mundial, abrange o desenvolvimento de métodos sistemáticos para auxiliar a tomada de decisão e vem sendo utilizada nas mais diversas áreas de produção e logística, de indústrias e organizações e serviços (BENITO et al., 2004, YANASSE et al., 2015). Inicialmente, o ensino dessas duas áreas fez parte de cursos como Administração e Engenharia de Produção. No entanto, surgiu uma oportunidade para que o ensino de técnicas mais apuradas com a ampliação do uso da Matemática também ocorresse em cursos de graduação e técnicos em Logística. Entre essas técnicas, cita-se a Teoria de Grafos, que estuda a relação entre elementos dentro de uma rede, através de estruturas matemáticas. Um grafo nada mais é do que um conjunto de pontos (que podem ser indivíduos, instalações, países etc.) que contenham relações entre si (CHALREO, 2016).

A Teoria dos Grafos estuda objetos combinatórios - os Grafos - que são modelos utilizados em muitos problemas de matemática, informática, engenharia, economia, sociologia e da indústria. Muitos desses problemas sobre grafos tornaram-se notáveis porque são um interessante desafio intelectual e porque têm importantes aplicações práticas. Tendo as suas raízes em jogos e recreações matemáticas, atribui-se a sua criação a Leonhard Euler (1707-1783), ao resolver o problema das pontes de Konigsberg, em 1736. No entanto, foram com os problemas acerca de fórmulas de estrutura de compostos químicos, que diversos matemáticos começaram a desenvolver tal teoria.

Os grafos são especialmente importantes na computação para a criação de algoritmos que determinem caminhos melhores, viáveis, encontrem e solucionem problemas e determinem meios de se resolver. Esse tipo de estudo é principalmente caracterizado por suas estruturas chamadas de grafos (do inglês, graphs) e não simplesmente gráficos, para não haver confusão com a área de estudo dos resultados da estatística, como boxplot, barras, dispersão, entre outros. Essas estruturas de grafos são compostas por um conjunto de objetos $G(V, A)$, onde $G$ é o conjunto desses objetos, V são os objetos em si ou simplesmente Vértices e $A$ são as arestas entre esses objetos (UHLMAMM, 2017). 
Atualmente, o uso de ferramentas de informática simplifica a compreensão e a otimização dos modelos. Dessa forma, é possível avaliar cenários de produção e distribuição com as suas respectivas consequências. Hoje, existe uma gama de softwares e programas com base em algoritmos matemáticos que são usados por profissionais da logística, objetivando o aumento dos lucros ou a redução dos custos. Mas como alunos da mesma turma que recebem o mesmo material didático e roteiros e possuem o mesmo professor podem apresentar resultados tão distintos? À luz desse questionamento, analisamos a produção de duas turmas do Ensino Superior em Logística, durante o semestre em que estudavam a disciplina de Pesquisa Operacional Aplicada à Logística, com foco na Teoria de Grafos, ministrada nos semestres de 2017/1 e 2017/2.

\section{Teoria da Atividade e a Modelagem Matemática: os pressupostos teóricos}

A Teoria Histórico-Cultural é a corrente psicológica que explica o desenvolvimento da mente humana com base nos princípios do materialismo dialético de Lev Semyonovich Vygostky (18961934). Há diferentes aportes trazidos por alguns teóricos dessa teoria, entre eles, Alexei Leontiev (1903-1979) e Vasily Davydov (1930-1998), para a compreensão das relações entre aprendizagem e ensino como processos cultural e historicamente mediados pela atividade humana.

Centrada na atividade humana, a Teoria Histórico-Cultural da Atividade (ou simplesmente Teoria da Atividade) surgiu como desdobramento da concepção histórico-cultural e foi desenvolvida por Leontiev e seus seguidores. O psicólogo soviético investigou a atividade a fim de demonstrar que o desenvolvimento psíquico humano encontra sua expressão na atividade psíquica como forma peculiar de atividade humana, "como um produto e um derivado da vida material, da vida externa, que se transforma em atividade da consciência" (LEONTIEV, 1978 apud GOLDER, 2002, p. 52). A atividade, nesse ponto, representa a ação humana que midiatiza a relação entre o homem, sujeito da atividade, e os objetos da realidade, dando a configuração da natureza humana. Portanto, o desenvolvimento da atividade psíquica tem sua origem nas relações sociais do indivíduo em seu contexto social e cultural.

As atividades humanas são formas de relação do homem com o mundo e são dirigidas por motivos e objetivos, agindo de forma intencional, por meio de ações planejadas (LEONTIEV, 1978). Nessa perspectiva, o contexto histórico-cultural de cada indivíduo influencia no seu aprendizado e, por consequência, na sua produção de conhecimento, além de influenciar a sua atuação profissional. Com o homem na vida adulta, por exemplo, o trabalho é atividade que guia seu desenvolvimento (LEONTIEV,1978). Ou seja, o contexto histórico-cultural do indivíduo influencia na sua produção de conhecimento e atuação profissional.

O conceito de atividade não se refere apenas a ações físicas desenvolvidas por um indivíduo, mas também a ações psíquicas conscientemente controladas, como a memorização ativa, o pensamento, o comportamento intencional. Desse modo, a atividade humana envolve ações externas e internas (LEONTIEV, 1978). Com isso, retomamos a discussão de Almeida e Brito (2005) 
para (i) atribuir sentido e construir significados em Matemática a partir de situações de ensino e aprendizagem que induzam relações entre a Matemática e a vida profissional de alunos do Ensino Superior e (ii) apontar como as atividades de Modelagem Matemática podem favorecer a aproximação da Matemática escolar com problemas do mundo do trabalho que serão vivenciados pelos alunos.

A Modelagem Matemática, no contexto da Educação Matemática, tem sido foco de muitos estudos nos últimos anos. Em termos genéricos, alguns autores apontam-na como a aplicação da Matemática em outras áreas de conhecimento, porém tal definição apresenta certa limitação teórica, dando a ideia de que qualquer atividade se caracteriza como Modelagem Matemática (BARBOSA, 2004). Outras vezes, a Modelagem Matemática é compreendida nos parâmetros da Matemática Aplicada, expressa em esquemas explicativos em que a principal dificuldade diz respeito aos quadros de referências postos pelo contexto escolar. Possivelmente, os esquemas explicativos, oriundos da Matemática Aplicada, apresentam-se como prescritivos sobre a atividade dos alunos, os quais são avaliados em termos do que falta para chegarem ao uso adequado deles (idem). Reforça-se, nesse sentido, a ideia do objetivo deste trabalho em evidenciar a necessidade de reflexão sistemática acerca da Modelagem Matemática sob a ótica escolar e no âmbito da Educação Matemática. Barbosa (2004, p. 2) afirma que "tal reflexão não significa uma separação da Matemática Aplicada, com a qual a Educação Matemática tem forte intersecção, mas sim a singularização do objeto no campo da Educação Matemática".

Embasando-nos em Silveira e Ribas (2004), podemos acreditar que os fatores que justificam a inclusão da Modelagem Matemática no currículo escolar, em específico de Educação Profissional, são: motivação, facilitação e envolvimento de alunos e professores no processo educativo; compreensão do papel sociocultural da Matemática e de sua utilização nas diferentes áreas do conhecimento, bem como de sua importância para formação profissional diante do mundo do trabalho; desenvolvimento de habilidades gerais de exploração/investigação; desenvolvimento do raciocínio, lógico e dedutivo em geral, que, consequentemente, implica a formação de cidadão crítico e transformador de sua realidade.

A partir do exposto, optamos por trabalhar com a Modelagem Matemática, pois a vimos como uma grande aliada da Educação Profissional, já que, ao desenvolver uma atividade dessa natureza, exploramos uma situação real; neste caso, a entrega das urnas vista como um problema de logística. Para desenvolver a atividade, utilizamos o terceiro caso apresentado por Barbosa (2004), com o propósito de aproximar o estudante de sua realidade profissional, uma vez que, neste caso, os alunos têm uma maior liberdade de escolher suas estratégias de resolução, assim como teriam na empresa que trabalharão após conclusão do curso. 


\section{Percurso metodológico}

No que se refere ao aspecto metodológico, realizamos um estudo qualitativo, do tipo exploratório (GIL, 2008). Iniciamos com um estudo bibliográfico sobre Teoria da Atividade, Pesquisa Operacional e suas aplicações no campo da Logística. Em seguida, nosso campo de investigação foi a disciplina de Pesquisa Operacional Aplicada à Logística, ofertada no Curso Superior de Tecnologia em Logística do IFES - Campus Viana, nos dois semestres de 2017, quando as turmas cursavam o penúltimo período. Delimitamos nossas análises na turma do primeiro semestre, que era composta por vinte e um estudantes, com idades entre 21 e 44 anos, tendo uma média etária de 28 anos.

Durante a disciplina, foi abordado o conteúdo de grafos, associado a situações da logística. Para resolução dos problemas, os alunos foram organizados em grupos e receberam fichas contendo o contexto de investigação e uma tarefa que motivaria a realização da modelagem. Ao longo do semestre, foram realizados quatro estudos de caso, "Modelagem em grafos de sistemas hub-and-spoke", "Entrega das urnas para eleição de Reitor no IFES", "Rede de distribuição de malotes do IFES" e "Planejamento do crossdocking no pátio de um centro de distribuição", em que os alunos modelavam e resolviam os problemas sempre nos mesmos grupos.

Nesse recorte, analisamos o estudo de caso denominado "Entrega das urnas para Eleição de Reitor do IFES". A proposta analisada partia do contexto eleitoral ocorrido em 2017 e propunha que os estudantes distribuíssem urnas nos campi da instituição, do modo econômico, e apresentassem um cronograma de entrega para que houvesse capacitação dos mesários com os funcionários do Tribunal Regional Eleitoral do Espírito Santo (TRE-ES).

\section{Reflexões sobre a experiência de ensino}

Ao analisar as produções dos estudantes, verificamos que alguns grupos entregaram trabalhos observando apenas a entrega das urnas, enquanto outros consideraram diversos fatores externos não solicitados, como jornada de trabalho dos servidores envolvidos, capacidade do portamalas do carro para carregar as urnas, volume de gasolina necessário, preço gasto com combustível e/ou pedágio etc. Ou seja, uma diferença considerável em relação aos demais grupos. Neste momento, a Teoria da Atividade traz uma justificativa para tal comportamento quando Leontiev afirma que uma atividade se distingue de outra pelo seu objeto e se realiza nas ações dirigidas a este objeto (LEONTIEV, 1978). Desse modo, a atividade humana não pode existir a não ser em forma de ações ou grupos de ações que lhes são correspondentes e na relação ativa do sujeito com o objeto por meio de ações, operações e tarefas, suscitadas por necessidades e motivos.

Um dos fatores que, de certa forma, está relacionado a essa intensidade de envolvimento na atividade é a motivação interna, influenciada pelo contexto histórico-cultural de cada indivíduo: a experiência laboral se manifesta em ações de trabalho; a atividade didática, em ações de 
aprendizagem; a atividade de comunicação, em ações de diálogo e assim por diante. Portanto, ainda que os alunos tenham recebido o mesmo material didático em sala de aula e desenvolvido as mesmas tarefas, houve diferentes estratégias de resolução para a mesma atividade. Discorreremos sobre a produção desse grupo que apresentou maior contribuição para a pesquisa devido à diferença em relação aos demais trabalhos e à quantidade de informações discriminadas na Modelagem Matemática.

Conforme anunciado, a tarefa propunha que os estudantes distribuíssem urnas nos campi da instituição, do modo econômico, e apresentassem um cronograma de entrega para que houvesse capacitação dos mesários com os funcionários do TRE-ES. O grupo analisado entregou um relatório descritivo das opções elegidas, por meio do qual foram perceptíveis as diferentes ferramentas utilizadas e opções realizadas em comparação ao restante da turma. Em um primeiro momento, os alunos elegeram duas rotas para entrega das urnas, cada qual com um veículo diferente, alegando ser o modo mais econômico analisado utilizando o algoritmo de Djikstra. Os mesmos sairiam de Vitória e partiriam com destino a diferentes campi para iniciar as respectivas rotas.

Figura 1 - Grafos e rotas elaborados pelos alunos do grupo com as rotas ótimas para entrega das urnas.

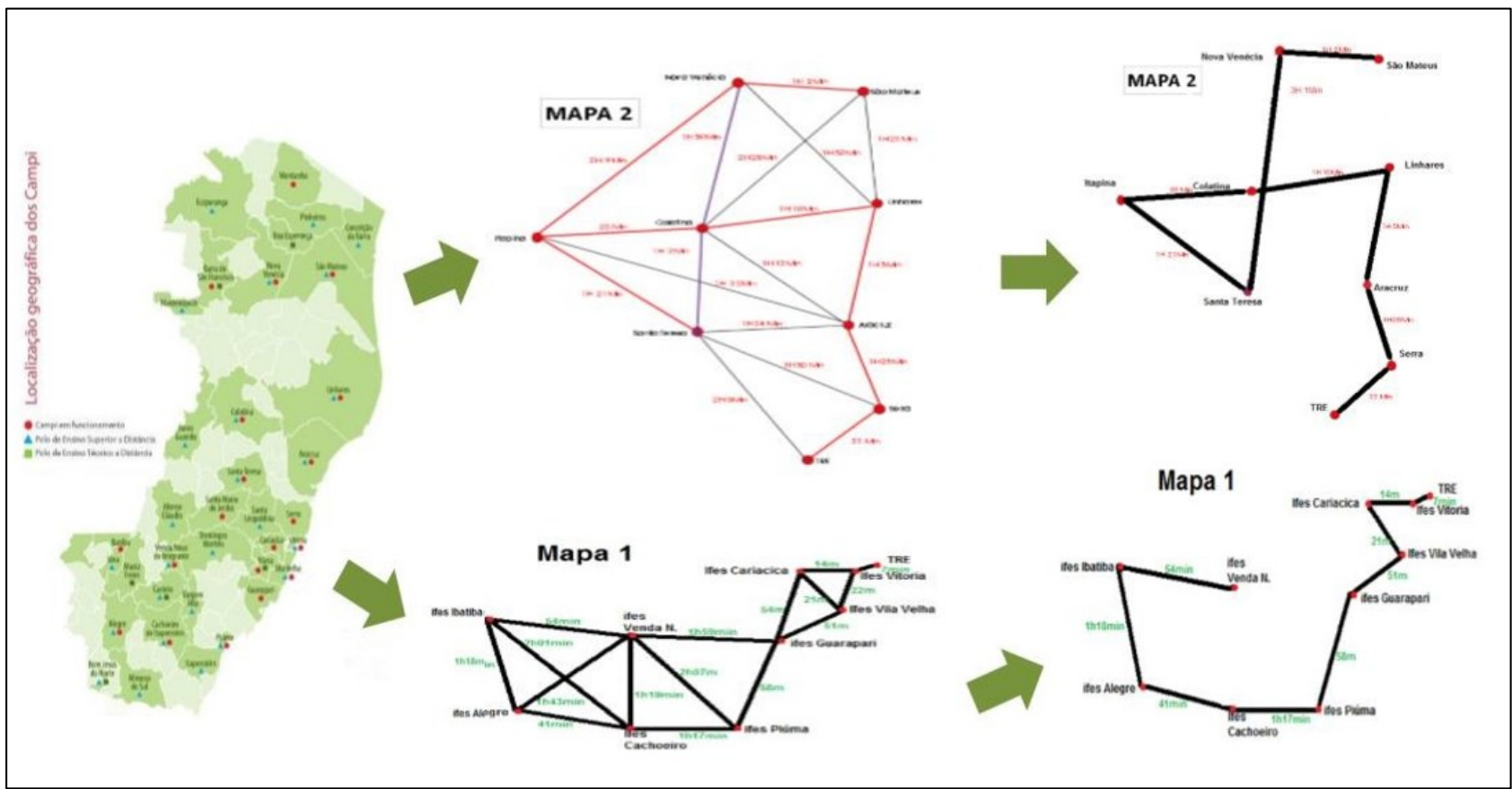

Fonte: Acervo dos autores (2017).

Os alunos ficaram livres para eleger o algoritmo que melhor thes atenderia para a resolução do problema, e a escolha inicial foi o de Djikstra, que soluciona problemas do caminho mais curto ou de custo mínimo entre dois vértices de um grafo. Nesse procedimento lógico, um vértice é escolhido como origem, e o algoritmo calcula o menor caminho desse vértice para todos os demais vértices do grafo até chegar ao destino. Ele é bastante simples e com um bom nível de otimização, 
pois parte de uma estimativa inicial para o custo mínimo e vai sucessivamente ajustando essa estimativa (BOAVENTURA NETTO, 2006).

Apesar de buscar otimizar o tempo de entrega, o processo de entrega de urnas para eleição de Reitor do IFES não pode ser resolvido somente com o algoritmo de Djikstra, uma vez que, por esse algoritmo, o menor caminho não precisa conter todos os vértices do grafo. Com isso, observamos que se trata de um Problema do Caixeiro-viajante, que consiste em determinar o caminho mais curto que passa exatamente uma vez por cada vértice e retorna ao vértice de partida. Esse é um problema NP-Completo e, embora tenham sido desenvolvidos bons algoritmos de aproximação para o PCV, não há, até o momento, a solução mais eficiente (BOAVENTURA NETTO, 2006). Desse modo, as heurísticas utilizadas pelos graduandos em Logística foram relacionadas ao método de construção de circuitos, em que os vértices vão sendo inseridos faseadamente, mediante certas condições e sem que exista qualquer modificação posterior à inserção definida pelo algoritmo. Em nosso caso, os circuitos foram elaborados através do método do vizinho mais próximo, ou seja, pela escolha da cidade não visitada e mais próxima do caixeiro (equipe do TREES), até que todas as cidades sejam visitadas.

Para eleger os pontos a serem percorridos em cada grafo, os estudantes lançaram mão da ferramenta de localização por satélite do Google Maps, de modo que a justificativa se tornasse a mais realista possível. Ao desconsiderar fatores externos, como congestionamentos e pedágios, e contemplar obrigatoriedades, como horário de almoço dos trabalhadores, os estudantes remeteramse à disciplina de Transportes e Seguros, como foi possível observar no extrato do relatório de modelagem produzido por um dos grupos acompanhados:

- O primeiro passo foi verificar as especificações apresentadas no texto que definem algumas questões que devem ser seguidas [...]

- Definimos que os 2 carros com as urnas partiriam no TRE, localizado em Vitória, seguindo um para o campus de Vitória e o outro para o campus da Serra. [...]

- A partir dessas definições decidimos usar o Google Maps para verificar as rotas [...].

- Ficou decidido pelo grupo que não levaríamos em consideração fatores externos como o trânsito, pausas nos trajetos para descansar, necessidades fisiológicas, paradas em semáforos, e nem nenhum imprevisto que possa atrasar a viagem [...]. Também desconsideramos 0 fato de a escolha da rota poder acarretar custos como pagamento de pedágios e gastos com locais para paradas noturnas. Essas decisões foram tomadas por acharmos que a definição da rota ficaria bem mais complexa, já que não possuímos essas informações e não temos tempo hábil para obtê-las.

- Consideramos parada para almoço com duração de 1 hora, porém sem horário definido previamente [..].

- As matérias aprendidas que de certa forma puderam ser utilizadas para realização da atividade proposta foi [sic] tecnologia da informação, já que utilizamos a ferramenta disponível e mais acessível para nós na internet que é o caso do Google Maps. E também utilizamos a matéria de Transportes e Seguros, para definirmos os fatores externos que poderiam 
influenciar na definição das rotas, e quais desses fatores nós consideraríamos e quais desconsideraríamos.

- A solução encontrada é viável, e muito possivelmente poderia ser realizada com sucesso, pois viabiliza o menor tempo de trajeto, ou o menor caminho, baseado no algoritmo de Dijkstra.

Relatório de Modelagem produzido por um dos grupos da pesquisa (2017, p. 1-2, grifos nossos).

A análise sobre o texto acima permitiu uma reflexão sobre a emancipação dos alunos quanto à resolução da atividade e comprometimento com o resultado final. Aqui foi apresentado somente um extrato do relatório, mas houve muitas outras pontuações feitas pelo grupo acompanhado e que, por sua vez, não foram realizadas pelos demais alunos da turma. Nesse caso, podemos citar a dúvida sobre como realizar o cálculo em relação ao tempo ou a distância entre as cidades, justificando que, por existir a necessidade de unir ao percurso o tempo gasto com treinamento, optaram pelo cálculo baseado no tempo.

Nesse trecho, ficou clara a importância da utilização da Modelagem Matemática para a resolução da atividade e, principalmente, da metodologia proposta por Barbosa (2004) quanto à liberdade dos alunos na formulação e solução de situações problema como o discutido, repercutindo no envolvimento dos alunos no processo educativo. Ademais, destaca-se a compreensão da relação da Matemática com as diferentes áreas do conhecimento, bem como de sua importância para formação profissional diante do mundo do trabalho. Também destacamos o desenvolvimento de habilidades gerais de exploração e investigação, do raciocínio lógico e dedutivo, que, consequentemente, implicam a formação de cidadão crítico e transformador de sua realidade.

\section{Algumas considerações}

Neste relato, nosso objetivo foi analisar um estudo de caso denominado "Entrega das urnas para Eleição de Reitor do IFES", realizado durante a disciplina de Pesquisa Operacional, ofertada no Curso Superior de Tecnologia em Logística do IFES - Campus Viana, nos dois semestres de 2017. A proposta teve o objetivo de trabalhar problemas logísticos reais que os alunos vivenciarão, como roteirização de veículos, analise de viabilidade de transporte etc., por meio da metodologia de Modelagem Matemática. Por meio da análise das produções de ambas as turmas, à luz da Teoria da Atividade, pôde-se observar a diferença de olhares sobre o mesmo problema entre os diferentes grupos da mesma disciplina. Isso reforça a ideia de Leontiev sobre a influência da motivação interna de cada indivíduo no resultado final de suas atividades.

Concluímos que essa experiência apontou para um ensino emancipador, pois a partir de um tema não-matemático, a eleição para Reitor, os alunos formularam e resolveram problemas, sendo responsáveis pela coleta de informações, simplificação das situações e realização da modelagem. Assim, esperamos que, com a emancipação dos alunos, alcancemos, por meio deles, a emancipação de toda a classe trabalhadora (SAVIANI, 2017). 


\section{Referências}

ALMEIDA, L. M. W. de; BRITO, D. dos S. Atividades de Modelagem Matemática: que sentido os alunos podem Ihe atribuir? Ciência \& Educação, v. 11, n. 3, p. 483-498, 2005.

ASSOCIAÇÃO BRASILEIRA DE ENGENHARIA DE PRODUÇÃO. Referências de conteúdos da engenharia de produção. Disponivel em:

<http://www.abepro.org.br/arquivos/websites/1/\%C3\%81reas $\% 20 \mathrm{da} \% 20$ Engenharia $\% 20 \mathrm{de} \% 20 \mathrm{Pr}$ odu\%C3\%A7\%C3\%A3o.pdf>. Acesso em: 12 out. 2011.

BARBOSA, J. C. Modelagem matemática: O que é? Por quê? Como?. Veritati, Salvador/BA, n. 4, p. 73-80, 2004.

BENITO, R. C. V.; SILVEIRA, C. A.; LAVRATTI, F. B. A otimização da logística de distribuição como diferencial competitivo. In: Mercolog. Porto Alegre/RS, 2004.

BOAVENTURA NETTO, P. O. Grafos: teoria, modelos, algoritmos. 5. ed. Edgard Blücher, 2006.

CHALRÉO, F. Teoria dos Grafos e análise de redes. 2016. Disponível em:

<http://www.ilos.com.br/web/teoria-dos-grafos-e-analise-de-redes/>. Acesso em: 05 jun. 2018.

FERRUZZI, E. C. A Modelagem Matemática como estratégia de ensino e aprendizagem do cálculo diferencial e integral nos cursos superiores de tecnologia. Dissertação (Mestrado em Engenharia de Produção e Sistemas) - Universidade Federal de Santa Catarina, Florianópolis, 2003.

GIL, A. C. Métodos e técnicas de pesquisa social. 6. ed. São Paulo: Atlas, 2008.

GOLDBARG, M. C.; LUNA, H. P. L. Otimização combinatória e programação linear. Rio de Janeiro, RJ: Elsevier, 2005.

GOLDER, M. (Org.). Leontiev e a Psicologia Histórico-Cultural - um homem em seu tempo. São Paulo: Grupo de Estudos e Pesquisa sobre Atividade Pedagógica; Xamã. 2002.

LEONTIEV, A. O desenvolvimento do psiquismo. Lisboa: Horizonte, 1978.

MOREIRA, D. A. Pesquisa Operacional: curso introdutório. São Paulo, SP: Tompson Learning, 2007.

SAVIANI, D. Prefácio. In: SÁ, L. C. e. Práticas pedagógicas na Educação Profissional:

experiências em cursos técnicos integrados ao ensino médio. Vitória, ES: Edifes, 2017. Disponível em: <http://edifes.ifes.edu.br/images/stories/praticas pedagogicas.pdf>. Acesso em: 25 mar. 2018.

SILVEIRA, J. C.; RIBAS, J. L. D. Discussões sobre Modelagem Matemática e o ensinoaprendizagem. 2004. Disponível em: <http://www.somatematica.com.br/artigos/a8>. Acesso em: 25 mar. 2004.

UHLMANN, E. A. Teoria dos Grafos: da teoria à aplicação computacional. Guarulhos: Instituto Siegen, 2017.

YANASSE, H. H.; ARENALES, M.; MORABITO, R.; ARMENTANO, V. A. Pesquisa Operacional. 2. ed. Elsevier, 2015. 
WILDE, D. J.; BEIGHTLER, C. S. Foundations of Optimization. Prentice Hall Inc., Upper Saddle River, 1967. 